\title{
Who is Missing in Planetary Science?: \\ A demographic study of the planetary science workforce
}

A white paper submitted to the Planetary Science and Astrobiology Decadal Survey 2023 - 2032

Primary Author: Edgard G. Rivera-Valentín ${ }^{1}$ (rivera-valentin@lpi.usra.edu);

${ }^{1}$ Lunar and Planetary Institute, Universities Space Research Association

Co-Authors: Julie Rathbun ${ }^{2}$, James Tuttle Keane ${ }^{3}$, Kennda Lynch ${ }^{1}$, Christina Richey ${ }^{3}$, Serina Diniega ${ }^{3}$, Janet Vertesi ${ }^{4}$

${ }^{1}$ Lunar and Planetary Institute, Universities Space Research Association

2Planetary Science Institute

3Jet Propulsion Laboratory, California Institute of Technology

${ }^{4}$ Department of Sociology, Princeton University

Endorsers: This white paper is submitted as part of a collaborative effort organized by the Equity, Diversity, and Inclusion Working Group (EDIWG), a cross Assessment Group (AG) committee.

Over $\underline{190}$ endorsers! Signatures can be found at the following link:

http://tiny.cc/EDI endorsers

Acknowledgements: A portion of the work described herein was carried out at the Lunar and Planetary Institute, which is operated by the Universities Space Research Association under a cooperative agreement with the Science Mission Directorate of NASA, and at the Jet Propulsion Laboratory, California Institute of Technology under a contract with NASA (80NM0018D0004). 


\section{Executive Summary}

The planetary science workforce surveys have shown that the demographics of the field are not representative of the national population (White et al., 2011; Hendrix et al., 2020), with this lack of diversity magnified in NASA mission teams (Rathbun et al., 2015; 2016; 2017). In order to identify trends in underrepresentation in planetary science and related fields and to inform diversity initiatives, we conducted a demographic analysis using the American Astronomical Society's Division of Planetary Sciences (DPS) 2020 workforce survey, as well as information from national sources. Our analysis considered race, ethnicity, and the representation of women by self-identification. Survey results identified four demographic groups that are underrepresented in planetary science. To a 95\% confidence level, women are underrepresented by $28.0 \% \pm 5.2 \%$, American Indian / Alaskan Native by $42.3 \% \pm 37.6 \%$, Latinx/ Hispanics by $76.3 \% \pm 5.7 \%$, and Black / African Americans by $91.6 \% \pm 4.0 \%$ with respect to the National Civilian Labor Force (NCLF) ${ }^{1}$. Although the field has made some improvements in regard to the representation of women, American Indian / Alaskan Natives, and Latinx / Hispanics, no change has occurred for Black / African Americans. Indeed, currently there is no significant difference between Black / African American student researchers and non-student researchers in planetary science. Similar analysis on the demographics of geoscience and physics doctoral graduates shows that no change in the representation of Black / African Americans has occurred over the last 18 years, which is in stark contrast to the overall increase of Black / African American doctoral graduates in Science and Engineering fields. Given these results, we provide a list of findings to motivate diversity initiatives; detailed recommendations follow in an accompanying white paper by Rathbun et al. However, we note that although demographic studies can help motivate diversity initiatives, they should not be the only discussion point in diversity, equity, and inclusion.

\section{Introduction}

Diversity and inclusiveness along multiple axes of representation is a social, business, and NASA imperative (e.g., Strategy 4.1, A Vision for Science Excellence ${ }^{2}$ ). Organizations that embrace diversity succeed in increasing creativity and innovation (e.g., Richard, 2000; Hong and Page, 2004). Additionally, research has shown that people from underrepresented minority groups typically draw new relationships between ideas and concepts and produce high rates of scientific novelty (e.g., AlShebli et al., 2018), but their work and scientific progress are systematically undervalued (Hofstra et al., 2020). Thus, scientific culture in the U.S. has at least two problems: it has both a diversity problem and an inclusion problem.

Diversity initiatives have attempted to bring the science community to parity with the national population. Although some initiatives have been effective, they have not all been inclusive of multiple minority groups. For example, the geosciences have seen a significant increase in the representation of women doctoral graduates, rising from $30 \%$ in 2000 to $49 \%$ in 2018; however, no improvement has occurred for Black / African American researchers (Bernard and Cooperdock, 2018). This is not due to a lack of interest! For example, both Black / African Americans and Latinx / Hispanics show interest in STEM comparable to White people

\footnotetext{
${ }^{1}$ https://www.bls.gov/cps/demographics.htm

${ }^{2}$ https://science.nasa.gov/science-pink/s3fs-public/atoms/files/2020-2024_Science.pdf
} 
(e.g., Crisp and Nora, 2012; Riegle-Crumb et al., 2019). Rather, the current lack of representation amongst scientists is a manifestation of a system with a long history of oppression. Another manifestation is in the wage gap. A survey of the higher education workforce found that for every dollar White men make, White women make $\$ 0.80$, men of color make \$0.72, and women of color make \$0.67 (McChesney, 2018).

In this white paper, we use results from the planetary science workforce surveys (White et al., 2011; Hendrix et al., 2020), as well as data from the National Center for Science and Engineering Statistics (NCSES) ${ }^{3}$, the U.S. Census Bureau, and the U.S. Bureau of Labor Statistics, to conduct a demographic analysis of the field. The intent of this white paper is to identify trends in underrepresentation in planetary science and related fields in order to motivate diversity initiatives for the coming decade. We acknowledge that most demographic surveys have limitations, such as the use of binary gender norms (e.g., see Strauss et al. for discussion), and that personal experiences are difficult to capture in such a quantitative manner. Indeed, although statistically grounded demographic studies can help provide direction for diversity initiatives, they should not be the only discussion point in diversity, equity, and inclusion.

\section{Workforce Demographics}

\subsection{Planetary Science Workforce}

Demographic data for race, ethnicity, and for women from the two workforce surveys are shown in Figure 1. The representation ratio is the percent representation of the demographic in the field with respect to the percent of that demographic in the NCLF for the same year. As a result, a ratio of 1 indicates parity, a value $>1$ indicates the demographic is represented above the national average, and a value $<1$ indicates underrepresentation. A property of this ratio is that it directly relates to the percent by which a group is underrepresented. Here, the margin of error is given to a 95\% confidence level (i.e., 2- $\sigma$ ) and represents the survey's ability to predict the demographics of the overall field. Additionally, the 2011 planetary workforce survey did not include student respondents, while the 2020 survey did; thus, to properly compare surveys we show results from the 2020 survey for student and non-student researchers separately.

In 2011, Latinx / Hispanics and Black / African Americans were underrepresented by 93.3\% $\pm 2.5 \%$ and $91.7 \% \pm 3.2 \%$ relative to the NCLF, respectively (i.e., a ratio of 0.07 and 0.08 ). Women were underrepresented by $48.1 \% \pm 3.4 \%$. No data was collected for American Indian / Alaskan Natives in the 2011 survey. Currently, nonbinary non-student researchers account for $0.7 \% \pm 0.4 \%$, men for $66.5 \% \pm 2.3 \%$, and women for $34.7 \% \pm 2.5 \%$ of planetary scientists; thus, women are underrepresented by $28 \% \pm 5.2 \%$. Over the past nine years the representation of women increased from $25 \% \pm 1.7 \%$ to $34.7 \% \pm 2.5 \%$. In terms of race and ethnicity, American Indian / Alaskan Natives are currently underrepresented by $42.3 \% \pm 37.6 \%$, Latinx / Hispanics by $76.3 \% \pm 5.7 \%$, and Black / African Americans by $91.6 \% \pm 4.0 \%$ with respect to the NCLF. We note, though, that American Indian / Alaskan Natives are historically undercounted in demographic surveys ${ }^{4}$ and thus their underrepresentation may be under estimated. The change in representation of Latinx / Hispanics from 2011 to 2020 is 3.0\% $\pm 1.0 \%$, which may be tracking the $2.9 \%$ national growth of the Latinx / Hispanic community during the same time period. However, no significant change has occurred for Black / African Americans $(0.1 \% \pm 0.6 \%)$.

\footnotetext{
${ }^{3}$ https://ncsesdata.nsf.gov/ids/sed

${ }^{4}$ http://blog.nativepartnership.org/the-importance-of-the-2020-census-for-native-americans/
} 


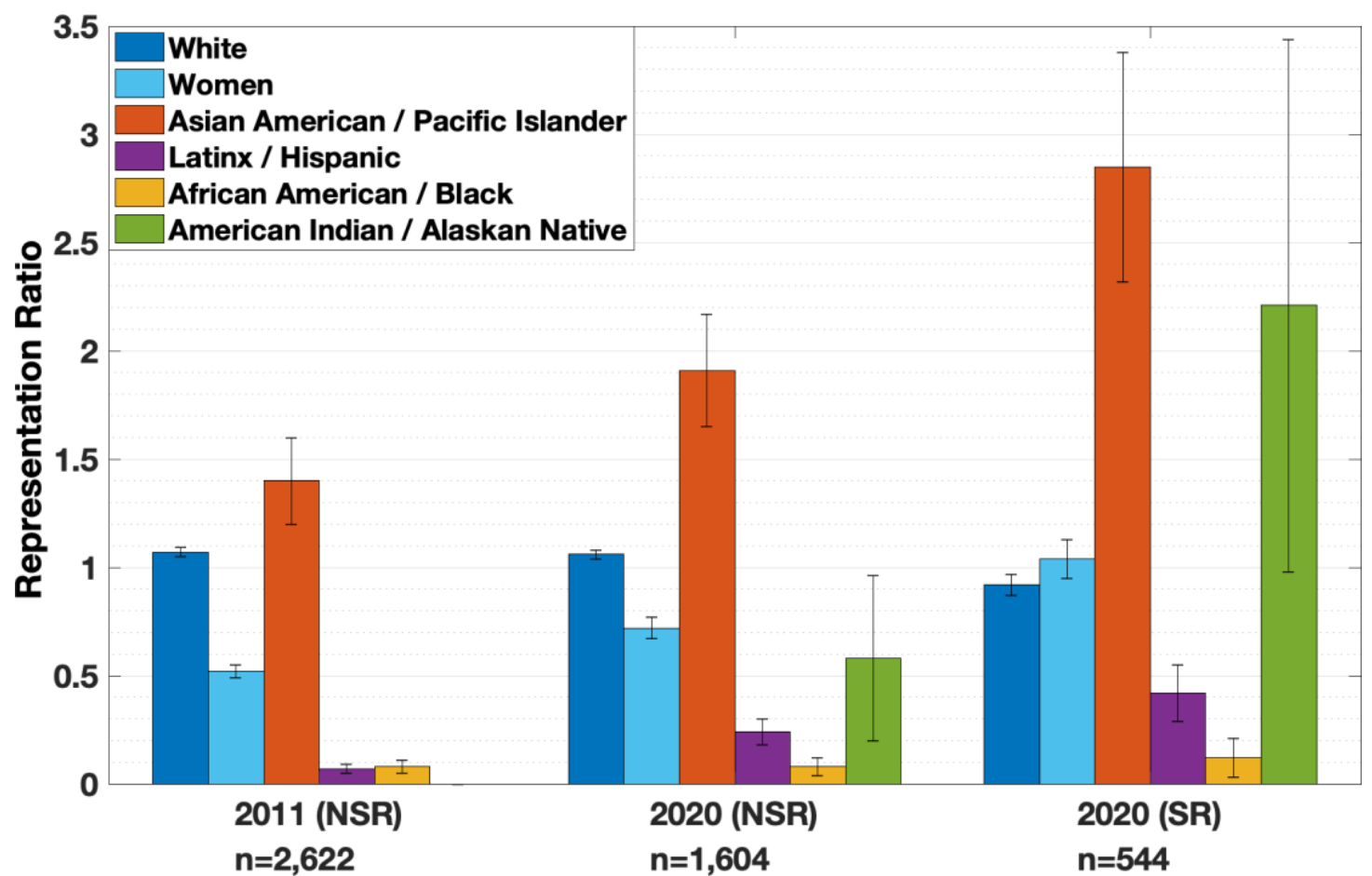

Figure 1: Representation ratio for the demographics surveyed in the 2011 and 2020 planetary science workforce surveys for non-student researchers (NSR) and student researchers (SR). The ratio is relative to the NCLF for the same year. The $x$-axis label includes the year of the survey and the total number of respondents. Error margins are to a 95\% confidence level. Note that no data was collected for American Indian / Alaskan Native in 2011.

White non-student researchers in 2011 were represented at $1.07 \pm 0.02$ and are currently at $1.06 \pm 0.02$ times their representation in the NCLF. Thus, they have not seen a significant change in their representation over the past decade. In 2011, Asian American / Pacific Islanders were represented at $1.4 \pm 0.2$ and currently are represented at $1.91 \pm 0.26$ times their representation in the NCLF. While Asian Americans / Pacific Islanders may not be underrepresented, this does not imply they do not face other challenges related to their identities, such as harassment. Additionally, the Asian American / Pacific Islanders demographic group is an aggregate category that consists of people who trace their roots to many different countries across East and Southeast Asia, the Indian subcontinent, and Pacific islands. Thus, this analysis is not well-suited to adequately identify representation across this diverse population.

One of the intents of diversity initiatives is to improve the demographics of the field. A good indicator of success is then the demographics of the student population. As seen in Figure 1 , there are three underrepresented demographic groups from non-student researchers (NSR) that have improved representation in the population of student researchers (SR). Of note, women comprise $50.3 \% \pm 4.2 \%$ and American Indian / Alaskan Natives comprise $2.2 \% \pm 1.2 \%$ of SR. Thus, within error, these two groups are represented near or above parity with respect to the NCLF. Not shown in Fig. 1, 2.6\% $\pm 1.4 \%$ of SR are nonbinary. In the SR population, Latinx / Hispanics are represented at $7.1 \% \pm 2.2 \%$, a difference of $3.1 \% \pm 2.4 \%$ compared to NSR; however, Latinx / Hispanics are still significantly underrepresented with respect to the NCLF among SR. Within error, there is no significant difference between Black / African American student researchers and non-student researchers. This indicates that diversity initiatives are not positively impacting the representation of Black / African American planetary scientists. 


\subsection{Physics and Geoscience Ph.D. Graduates}

The workforce surveys indicated that planetary scientists generally earned their degrees in physics or geoscience. Thus, we studied the demographics of physics and geoscience doctoral graduates (Fig. 2) to understand the potential role of the "pipeline", specifically for women, Latinx / Hispanic, and Black / African Americans. We use a weighted least squares fit to find the percent change over time. Since 2000, geosciences have seen an increase in the representation of women by $0.88 \% \pm 0.18 \%$ per year, while representation in physics has only grown by $0.33 \% \pm 0.15 \%$ per year. During the same time, Latinx / Hispanic representation in geology and physics has increased by $0.21 \% \pm 0.05 \%$ and $0.13 \% \pm 0.06 \%$, which is below the national growth of the Latinx / Hispanic community ( $0.32 \%$ per year). However, no change has occurred for Black / African Americans in either field over the last 18 years $(0.03 \% \pm 0.04 \%)$. This is in stark contrast to the overall increase of Black / African American doctoral graduates in Science and Engineering (0.09\% $\pm 0.02 \%$ per year) .

Further underrepresentation occurs for women of color. Since 2000, Latinx / Hispanic women have accounted for $48.7 \% \pm 4.3 \%$ and $19.6 \% \pm 2.9 \%$ of the doctoral degrees earned by Latinx / Hispanics in geology and physics respectively, and thus are underrepresented in physics with respect to Latinx men. Black / African American women are underrepresented in both fields with respect to Black / African American men as they account for $23.3 \% \pm 4.6 \%$ and $34.3 \% \pm 6.9 \%$ of the doctoral degrees earned by Black / African Americans in geology and physics, respectively. Women of color face additional challenges, such as increased harassment and discrimination (e.g., Clancy et al., 2017).

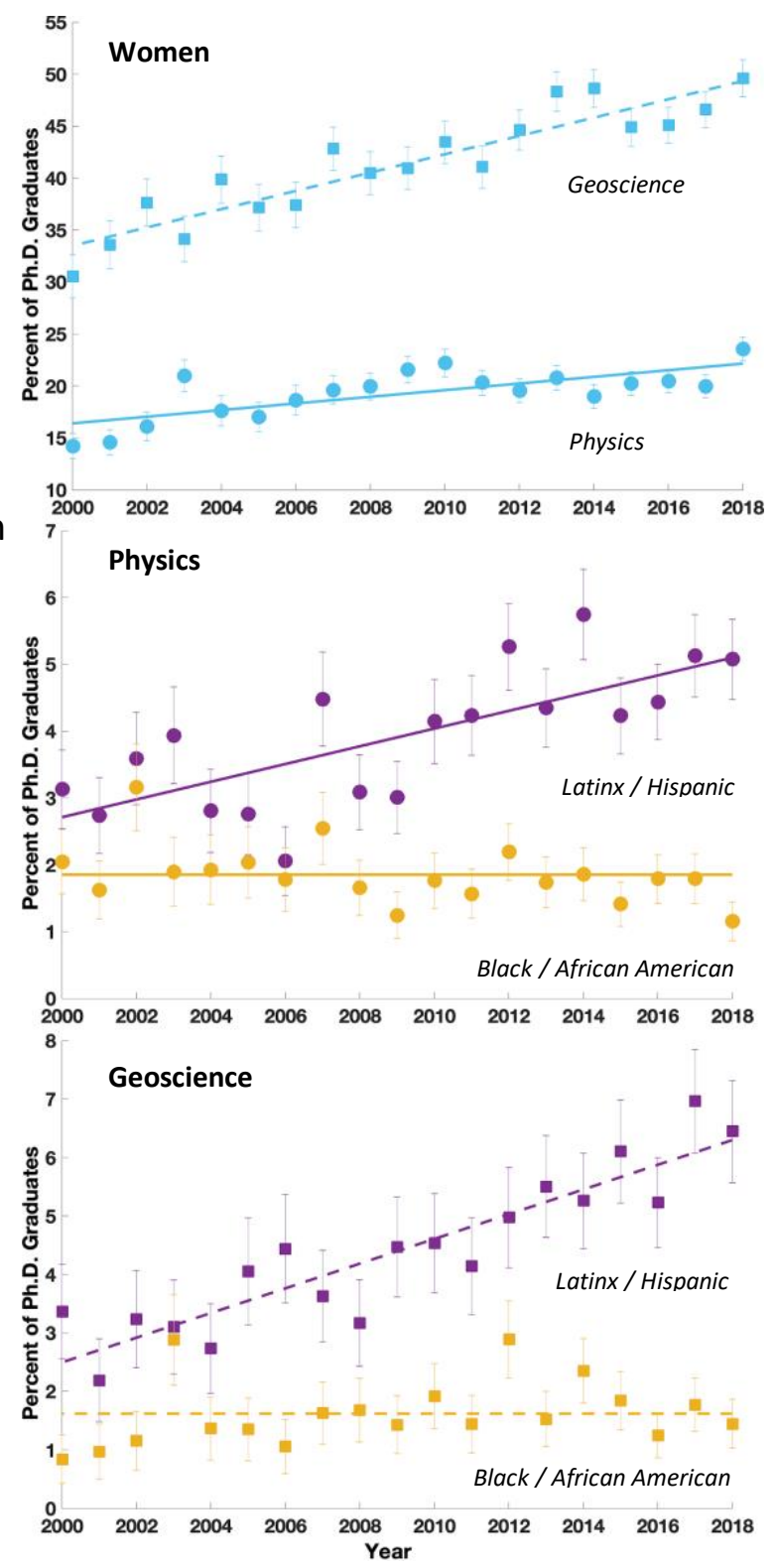

Figure 2: Percent representation of doctoral graduates for (top) women in physics (circles and solid line) and geoscience (squares and dashed line), and (bottom) Black / African American and Latinx / Hispanic (following the color coding from Figure 1). The lines are weighted least squares fit to the data from NCSES.

\subsection{NASA Missions}

Rathbun et al. $(2015 ; 2016 ; 2017)$ analyzed participation on the science teams of NASA spacecraft missions. They determined the percentage of women participating in the original science teams of 26 NASA robotic missions over a 41-year period. They found that from 2001 through 2015 the average rate of participation of women remained constant at about $15 \%$. 
Thus, women on spacecraft teams were underrepresented by $40 \%$ relative to the planetary science workforce. Although Rathbun et al. $(2015 ; 2016 ; 2017)$ studied the participation of women on planetary spacecraft science teams, they did not analyze other demographics, such as race. Demographic surveys of NASA mission teams are needed in order to inform policies that dismantle systemic barriers to inclusion of underrepresented groups. Rathbun et al. (2020) demonstrated that change is possible. They noted that the science team for Dragonfly, which was selected after NASA added a statement valuing diversity to the call for proposals, is composed of $42 \%$ women, a dramatic improvement from the earlier $15 \%$ women.

\subsection{NASA Workforce}

Using NASA Workforce data from the FY2018 MD-715 Report ${ }^{5}$, in Figure 3 we show the demographics of the overall NASA employee workforce and the NASA employees in science and engineering positions. In the overall NASA workforce, women are underrepresented by $29.5 \% \pm$ $1.5 \%$, Latinx / Hispanic by $55.3 \% \pm 2.4 \%$, and Black / African Americans by $10.8 \% \pm 3.7 \%$ with respect to the NCLF. The level of underrepresentation worsens in the Science and Engineering workforce where women are underrepresented by $51.7 \% \pm 1.6 \%$, Black / African Americans by $52.3 \% \pm 3.4 \%$, and Latinx / Hispanic by $57.6 \% \pm 2.8 \%$. On average, in both workforces, Asian Americans / Pacific Islanders are represented at $1.3 \pm 0.1$ times their representation in the NCLF. While the overall NASA workforce results are inconclusive for American Indian / Alaskan Natives as the representation ratio is $1.0 \pm 0.2$, in the Science and Engineering workforce they are underrepresented by $20.0 \% \pm 16.7 \%$.

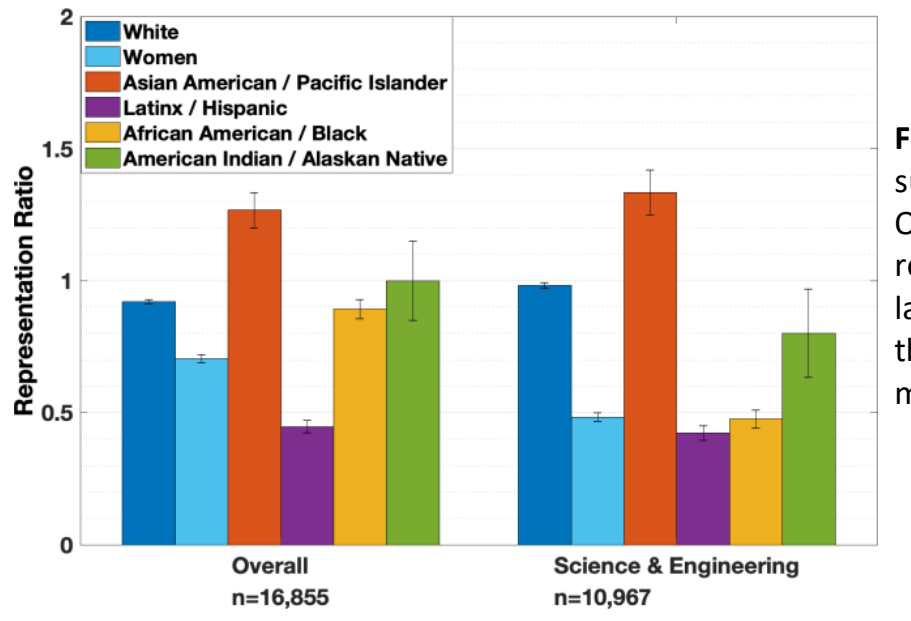

Figure 3: Representation ratio for the demographics surveyed by the NASA Office of Diversity and Equal Opportunity in their FY 2018 report. The ratio is relative to the NCLF for the same year. The $x$-axis label includes the studied employee population and the total number of participants in the survey. Error margins are to a $95 \%$ confidence level.

\subsection{Planetary Science Workforce by Job Type}

By cross correlating the answers to the 2020 DPS workforce survey, we also analyzed the demographics of the field by job type, which included tenured, tenure-track, hard money, soft money, and postdoctoral positions. The results are shown in Figure 4 with respect to the representation ratio of White, women, Asian American / Pacific Islander, and Underrepresented Racial Minority (URM) researchers. Due to the small number of responses per category by demographic group, Latinx / Hispanic, Black / African American, and American Indian / Alaskan Native demographics were aggregated to the URM category to preserve respondent privacy.

\footnotetext{
${ }^{5}$ https://www.nasa.gov/sites/default/files/atoms/files/2018 nasa md 715 report 5-15-2019 tagged.pdf
} 


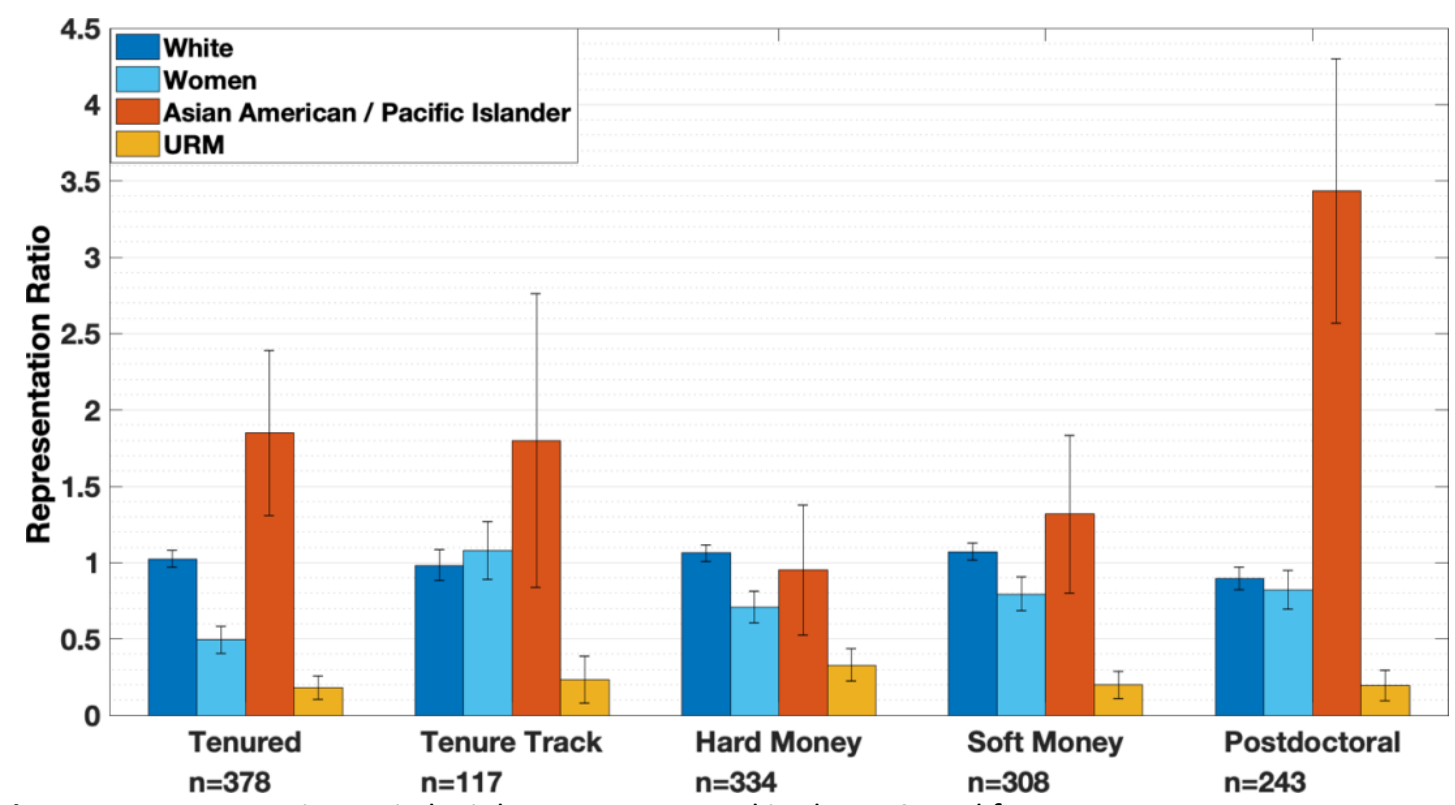

Figure 4: Representation ratio by job type as surveyed in the DPS workforce survey.

On average across all job types, URMs are represented at $7 \% \pm 1.9 \%$. Given that URMs comprise $31 \%$ of the NCLF, then Latinx / Hispanics, Black / African Americans, and American Indian / Alaskan Natives are underrepresented in all job types by $77.3 \% \pm 6.0 \%$. No statistical differences between job types exists for URM. The same is true for White researchers, which are near or above parity in all positions. The two demographic groups with differences with respect to job type are Asian American / Pacific Islanders and women. Asian American / Pacific Islanders are $20.6 \% \pm 6.2 \%$ of postdoctoral positions, while on average they are $6.8 \% \pm 2.0 \%$ of hard and soft money researchers and $11 \% \pm 3.3 \%$ of researchers in tenured and tenure-track positions. Women are represented at $52.1 \% \pm 9.1 \%$ in tenure-track positions, compared to all other positions where women are represented at an average of $33.9 \% \pm 2.6 \%$.

\section{Key Findings}

Here, we summarize our key findings. For detailed recommendations, please refer to the companion paper by Rathbun et al. Additional recommendations can be found in the white paper by Schmidt et al. In this white paper, we considered race, ethnicity, and the representation of women by self-identification. For a discussion of diversity and inclusion across other axes of representation, as well as specific and actionable recommendations, see white papers by, e.g., Milazzo et al., Piatek et al., Strauss et al., and Vander Kaaden et al.

Finding: Black / African Americans are significantly underrepresented in the planetary science workforce, including among student researchers in planetary science, geoscience, and physics. Over the past decades, Black / African Americans have seen no improvement in representation. Finding: Latinx / Hispanics are underrepresented in the planetary science workforce, including among student researchers in planetary science, geoscience, and physics. While their representation has improved, it may only be tracking the population's national growth. Finding: American Indian / Alaskan Natives are underrepresented in the planetary science workforce and NASA science and engineering workforce. Diversity initiatives may be positively impacting American Indian / Alaskan Natives since they may be represented near or above parity in the group of planetary science student researchers and the overall NASA workforce. 
Finding: Women are currently underrepresented in the planetary science workforce and physics Ph.D. programs. Diversity initiatives may have positively impacted women over the past decades. The representation of women is at parity in geoscience Ph.D. programs, planetary science tenure-track positions, and in the group of planetary science student researchers. Finding: Given the little to no change of the Black / African American and Latinx / Hispanic populations in planetary science and related fields, the representation growth of women over the past years may be primarily attributed to White women.

Finding: Women of color face additional barriers. For example, Black / African American women are underrepresented compared to Black / African American men in physics and geoscience Ph.D. programs, and Latinx / Hispanic women are underrepresented compared to Latinx/ Hispanic men in physics Ph.D. programs.

Finding: The underrepresentation of women is magnified on the science teams of NASA spacecraft missions. This suggests that even once a member of an underrepresented group becomes a professional scientist, there are still barriers to their progress. There is little data about representation along other axes, such as race and ethnicity, in science teams.

Here we highlight two recommendations from the white paper by Rathbun et al., but encourage the reader to refer to that white paper for detailed actionable recommendations. Recommendation: The decadal survey should explicitly recognize the underrepresentation of Black / African Americans, Latinx / Hispanics, and American Indian / Alaskan Natives in the current planetary science workforce. The decadal survey should note that diversity initiatives have not affected the representation of Black / African Americans over the past decades. Recommendation: We recommend that NASA implements a plan and directs funding for demographic studies of spacecraft mission teams and grants proposed and awarded. In particular, such studies must account for multiple groups (e.g., gender, race, ethnicity). The results should then be used to inform and motivate targeted diversity and inclusion activities.

\section{References}

AlShebli, B.K., Rahwan, T., \& Woon, W.L. (2018). Nature Communications, 9, 5163.

Bernard, R.E. \& Cooperdock, E.H.G. (2018). Nature Geoscience, 11, 292-295.

Crisp, G. \& Nora, A. (2012). White paper for the Hispanic Association of Colleges and Universities.

Hendrix, A.R. et al. (2020). Lunar and Planetary Science Conference, id.2813.

Hofstra, B. et al. (2020). Proceedings of the National Academy of Sciences, 117 (17), 9284-9291.

Hong, L. \& Page. S.E. (2004). Proceedings of the National Academy of Sciences, 101 (46), 1638516389.

McChesney, J. (2018). Representation and Pay of Women of Color in the Higher Education Workforce.

Rathbun, J.A., et al. (2015). American Astronomical Society, Division for Planetary Science, id.312.01.

Rathbun, J.A., et al. (2016). American Astronomical Society, Division for Planetary Science, id.332.01. Rathbun, J.A. (2017). Nature Astronomy, 1, 0148.

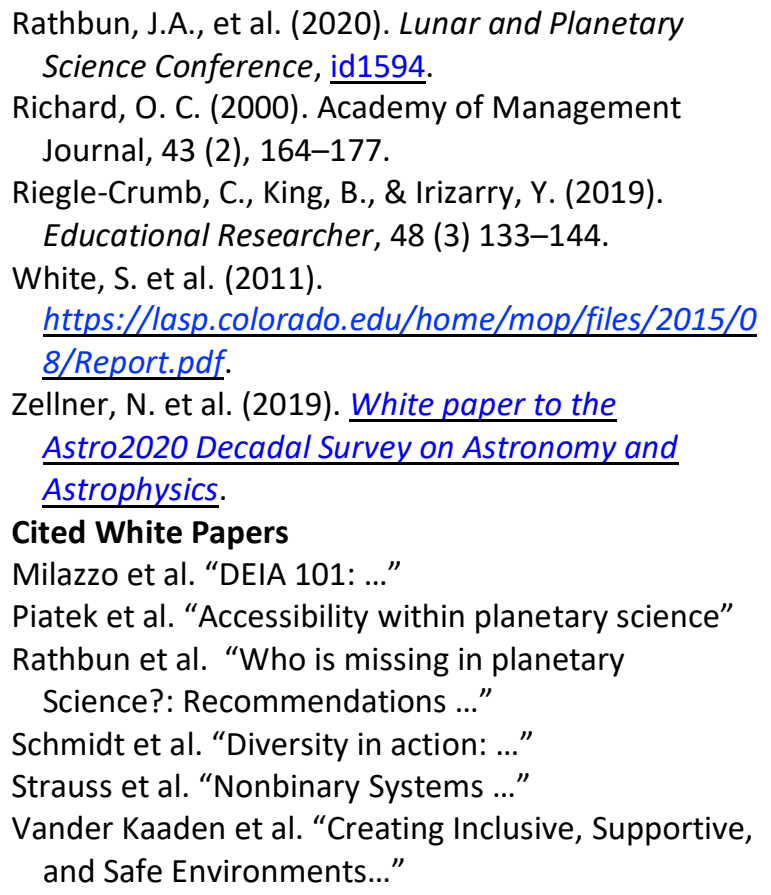

